\title{
L'évolution des paysages de référence, un angle mort dans la gouvernance des paysages?
}

L'exemple du mont Lozère

Is the evolution of baseline landscapes a blind spot in the landscape

governance?

The example of Mont Lozere, France

Clémence Moreau, Cécile Barnaud et Raphaël Mathevet

\section{OpenEdition \\ Journals}

Édition électronique

URL : http://journals.openedition.org/developpementdurable/14341

DOI : 10.4000/developpementdurable.14341

ISSN : 1772-9971

Éditeur

Association DD\&T

Référence électronique

Clémence Moreau, Cécile Barnaud et Raphaël Mathevet, «L'évolution des paysages de référence, un angle mort dans la gouvernance des paysages ? », Développement durable et territoires [En ligne], Vol.

10, n² I Juillet 2019, mis en ligne le 15 juillet 2019, consulté le 16 mars 2020. URL : http:// journals.openedition.org/developpementdurable/14341 ; DOI : https://doi.org/10.4000/ developpementdurable.1434

Ce document a été généré automatiquement le 16 mars 2020. 


\section{L'évolution des paysages de référence, un angle mort dans la gouvernance des paysages?}

L'exemple du mont Lozère

Is the evolution of baseline landscapes a blind spot in the landscape

governance?

The example of Mont Lozere, France

Clémence Moreau, Cécile Barnaud et Raphaël Mathevet

1 La France a connu après la Seconde Guerre mondiale d'importants bouleversements paysagers, avec l'intensification des pratiques sur les terres les plus productives et la déprise agricole sur les terres marginalisées (Perrier-Cornet, 2002; Hervieu et Purseigle, 2013).

2 L'émergence du paysage comme objet de gouvernance a connu plusieurs jalons. Les années 1970 voient l'affirmation du paysagisme dans l'aménagement du territoire (Luginbühl, 2007), mais il faut attendre la loi paysage de 1993 pour observer l'émergence de politiques paysagères portées par les collectivités territoriales (Labat et Aggeri, 2013), puis l'adoption de la Convention européenne sur le paysage en 2000, pour que le paysage soit reconnu à l'échelle européenne (CE 2000). Dans les aires protégées, en 2006, la loi de réforme réaffirme que les parcs nationaux doivent protéger non seulement les «milieux naturels », mais aussi le "paysage » (Filoche, 2007; Larrère et al., 2009). Dans le champ scientifique, le paysage connait à partir de 1970 un regain d'intérêt: il est au centre de divers courants de pensée comme le paysagisme (Donadieu, 2009), la géographie culturelle (Berque, 1994) ou l'écologie du paysage (Burellier et Baudry, 1999). Nous nous référons dans cet article au paysage comme construction sociale, c'est-à-dire comme le produit des perceptions individuelles et d'une représentation collective, qui transforme un territoire perçu en paysage, par le biais de normes et de discours, qui évoluent dans le temps (Sgard, 2011). 
3 Dans ce champ de réflexion, une thématique s'impose: celle de la fermeture du paysage. À partir des années 1960, la déprise agricole et le déclin démographique des campagnes, considérés jusqu'alors comme une condition de la modernisation agricole, inquiètent : ils seraient une dégradation esthétique, le signe de la fin de l'agriculture dans les zones défavorisées, voire de la mort sociale des campagnes (Le Floch et al., 2005).

4 Ces études ont révélé un postulat souvent implicite : l'existence d'un état de référence, celui des paysages ouverts, qui a une valeur descriptive et normative. De fait, la fermeture des paysages est considérée comme problématique, car elle induit un décalage entre l'état de référence et le paysage en voie de transformation (Floch et Devanne, 2003). La désignation des paysages ouverts comme paysage de référence résulte d'une construction historique, qui tient à la fois de l'avancée des connaissances scientifiques et de l'évolution des normes (Marty et Lepart, 2001). Par exemple, sur les Causses, un renversement normatif a lieu dans les années 1990: "On passe d'un consensus autour de la forêt à un consensus autour des milieux ouverts » (Lepart et al., 2000 : 22). Ce consensus autour des paysages ouverts met en avant la fonction potentiellement fédératrice du paysage dans la gouvernance des territoires (Davodeau, 2007 ; Sgard, 2011).

5 Mais que se passe-t-il lorsque les paysages ouverts changent, notamment sous l'effet de l'évolution des pratiques agricoles? Si les jeux d'acteurs liés à la fermeture des paysages font l'objet de nombreuses études (Friedberg et al., 2000 ; Deuffic, 2005 ; Le Floch et al., 2005), peu d'études traitent des jeux d'acteurs dans ce moment particulier d'évolution des paysages ouverts. À partir de deux cas d'étude alpins, Peyrache-Gadeau et Perron (2010) montrent que l'évolution des paysages ouverts sous l'effet de nouvelles pratiques peut fragiliser la fonction fédératrice du paysage, opérant par là une phase de déconstruction d'une représentation stabilisée $d u$ paysage. Une phase de reproblématisation du projet territorial semble alors nécessaire, dans laquelle le paysage peut constituer une ressource mobilisée dans l'élaboration du projet de développement. Or, l'approche du paysage-ressource présente selon Sgard des limites : il objective le paysage, par le biais d'un discours commun porté par les collectivités, ce qui peut être réducteur. Guisepelli (2004) mobilise quant à lui la notion de modèle paysager pour expliquer comment l'évolution des paysages ouverts découle de l'évolution des pratiques, elles-mêmes sous-tendues par différentes représentations, qui se traduisent par différents modèles de développement. Cependant, cette approche ne permet pas forcément d'aborder la question de la temporalité des paysages de référence. Finalement, l'enjeu est certainement d'aborder les paysages de référence comme un « objet insaisissable et mouvant » (Sgard, $2011: 163)$ pour mettre en lumière les jeux d'acteurs: "insaisissable ", car les paysages de référence ne doivent pas être objectivés, mais donner à voir les diverses représentations, et "mouvant», car leur caractère dynamique doit être central dans l'analyse.

6 L'objectif de cet article est de répondre à cet enjeu en mobilisant la notion de service écosystémique (SE), dans une approche ancrée en géographie sociale. Par SE, on entend les biens et les services que les hommes peuvent tirer des écosystèmes, directement ou indirectement, pour assurer leur bien-être (MEA 2005). Les SE relèvent de trois catégories: les SE d'approvisionnement comme les produits agricoles, les SE de régulation comme la pollinisation, les SE culturels comme la valeur esthétique des paysages. Nous mobilisons aussi la notion de disservice écosystémique, définie comme 
des biens et des services issus de l'écosystème qui diminuent ou dégradent le bien-être humain, comme les dégâts des sangliers sur les cultures (Shackleton et al., 2016).

7 Certaines études ont considéré le concept de SE (ou celui, très proche, d'aménité) peu approprié pour saisir l'objet paysage, car il induirait une convergence de regards et définirait le paysage comme un bien économique (Peyrache-Gadeau et Perron, 2010; Sgard, 2011). Notre point de vue est différent. Loin d'adopter une approche économique, nous considérons que la notion de SE peut aider à appréhender le paysage comme construction sociale et à mettre en lumière les jeux d'acteurs. Plusieurs auteurs ont mobilisé la notion de SE pour décrypter la diversité des représentations autour d'un enjeu donné (Lamarque et al., 2011; Felipe-Lucia et al., 2015 ; Barnaud et al., 2015), ou pour caractériser les relations sociales associées à cet enjeu (Felipe-Lucia et al., 2015 ; Berbés-Blázquez et al., 2016; Barnaud et al., 2018). Finalement, dans ce travail, nous faisons l'hypothèse que mobiliser la notion de SE peut mettre en évidence la façon dont les jeux d'acteurs se redessinent quand les paysages de référence évoluent.

8 Nous appuierons cette réflexion sur l'étude du cas emblématique du mont Lozère, dans lequel les paysages ouverts évoluent alors même qu'ils sont reconnus comme les paysages de référence par différents statuts de protection.

9 Dans un premier temps, nous présentons notre terrain et la problématique des changements paysagers en cours, puis le cadre conceptuel et les méthodes déployées. Dans un deuxième temps, nous analysons, via la grille de lecture des SE, les intérêts des acteurs concernés, leur relation à la nature et les institutions qui participent du changement et de la régulation des interactions entre les $\mathrm{SE}$ associées aux problématiques de dérochage et de retournement de prairies. Enfin, nous discutons brièvement comment les évolutions constatées invitent à repenser le caractère subjectif et dynamique des paysages de référence, ainsi que leur rôle dans la gouvernance des paysages.

\section{Concepts et méthodes}

\subsection{Site d'étude : le mont Lozère et ses paysages ouverts}

10 Le mont Lozère est un massif granitique des Cévennes culminant à 1699 mètres. Les paysages de crêtes, ponctués de chaos de granite, sont le point d'aboutissement des transhumances ovines. Sur les pentes, les forêts (hêtraies/sapinières ou plantations de résineux) et les parcelles agricoles (parcours, prés de fauche), majoritairement dédiées à l'élevage bovin, se partagent l'espace (PNC 2013).

11 Le cas du mont Lozère est fort pertinent pour alimenter la réflexion sur les jeux d'acteurs liés à l'évolution des paysages de référence. Le territoire est soumis à une forte croissance du couvert forestier ( $+34 \%$ entre 1970 et 1999, Corine Land Cover). La lutte contre la fermeture des paysages a été structurante dans la gouvernance des paysages, et les acteurs locaux comme les organismes de protection ont bâti leur légitimité d'action sur le maintien des paysages ouverts, notamment le Parc national des Cévennes (PNC), créé en 1970, et la réserve de biosphère du programme Man and Biosphere de l'Unesco (1984). Ces actions ont conduit à l'inscription de ces paysages représentatifs de l'agropastoralisme méditerranéen au patrimoine mondial de l'Unesco (2011). 
ces paysages ouverts évoluent, notamment avec l'augmentation du dérochage et du retournement de prairies. Le dérochage consiste à enlever les rochers qui affleurent pour aplanir le terrain et faciliter l'exploitation des parcelles. Cette pratique, ancienne sur le mont Lozère, s'est accélérée et transformée ces dernières années avec la mécanisation: d'une part, le dérochage est nécessaire pour labourer et faucher les parcelles, d'autre part, les pelles mécaniques permettent d'ôter des cailloux bien plus gros. Par retournement de prairies, on entend le labour d'une prairie permanente ou d'un parcours, devenant alors une prairie temporaire, qui sera semée et labourée régulièrement. L'augmentation récente des pratiques de dérochage et de retournement de prairie induit un décalage entre d'une part le paysage de référence, le modèle agropastoral où les parcours dominent, qui est encouragé par le PNC et l'Unesco, et le paysage en voie de transformation d'autre part, où la composante agricole, notamment les prairies temporaires, est plus importante. Le consensus autour des paysages ouverts s'en trouve bouleversé : des divergences de points de vue apparaissent, et le jeu d'acteurs est considérablement remanié.

\subsection{Cadre conceptuel}

13 Afin d'analyser les jeux d'acteurs, nous mobilisons la notion de $\mathrm{SE}$, et plus spécifiquement les interactions entre SE. Bennett et al. (2009) ont montré que les SE sont en antagonisme ou en synergie et que les changements sur les écosystèmes peuvent affecter simultanément plusieurs SE. Nous étudions ici les interactions entre SE selon une approche développée par Barnaud et al. (2018) qui déploient l'idée que révéler les interactions entre SE peut être un moyen de mettre en évidence des interdépendances sociales qui n'étaient pas forcément visibles ou explicites, voire volontairement cachées par certains groupes d'acteurs. Il s'agit d'interdépendances entre différents types d'acteurs: les fournisseurs de SE, comme les éleveurs qui façonnent des paysages, les bénéficiaires de SE comme les touristes, et les acteurs intermédiaires, comme le PNC, qui influencent indirectement la production de SE. En particulier, les antagonismes entre SE conduisent à des conflits d'intérêts entre bénéficiaires de $\mathrm{SE}$ et sont le siège de choix sociaux qui ne sont pas toujours explicités ni collectivement négociés (Barnaud et Antona, 2014). Nous proposons ici d'utiliser ce cadre en l'associant à l'approche des « trois I » en sciences politiques (Heclo, 1994 ; Hall, 1997) qui permet d'étudier les liens entre trois dimensions habituellement cloisonnées dans l'analyse en sciences sociales : les intérêts, les institutions et les idées. 
Figure 1. Le cadre conceptuel mobilisé dans cette étude

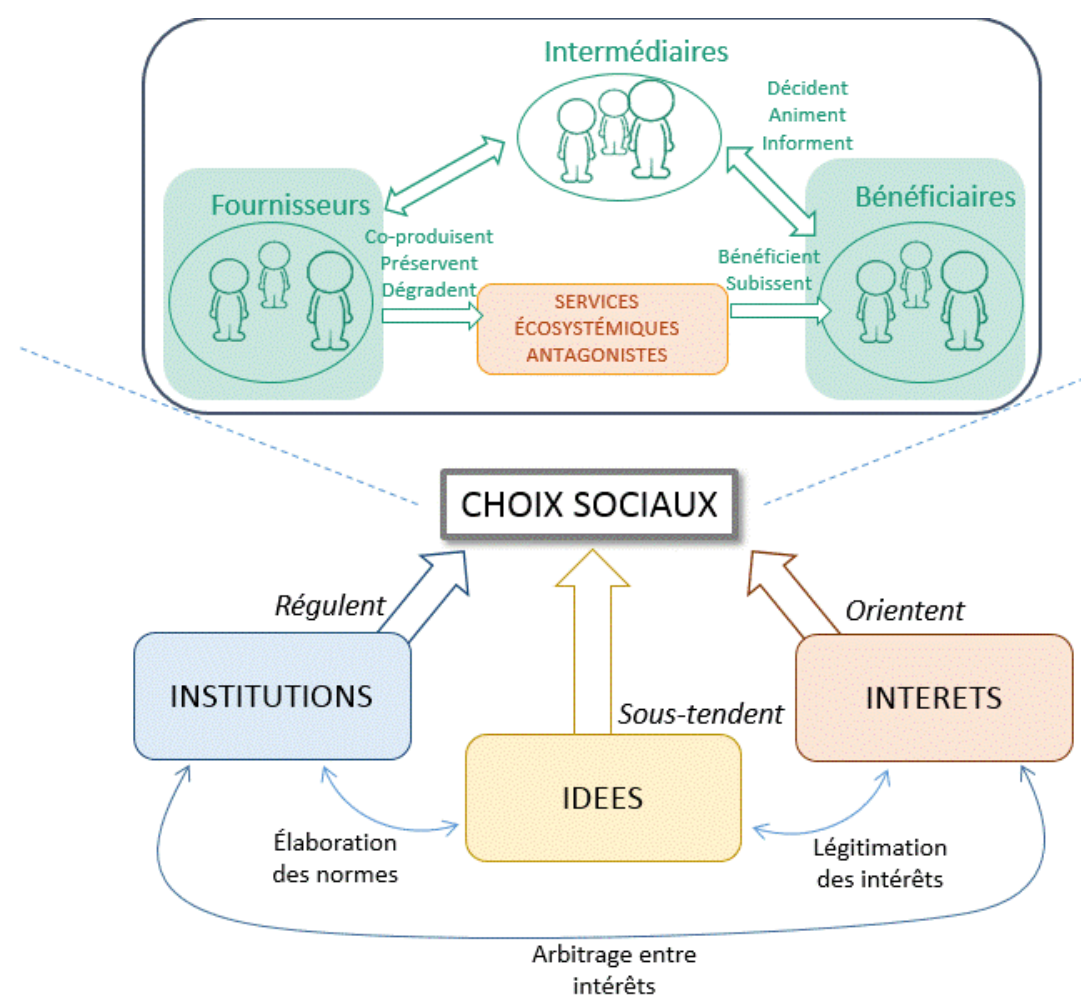

La notion d'intérêt postule que les acteurs font des choix en suivant une certaine rationalité, en fonction de leurs intérêts individuels, qu'ils cherchent à maintenir, défendre ou optimiser. Cette analyse éclaire ainsi les stratégies individuelles, mais aussi les dynamiques collectives de confrontation des intérêts à travers les conflits et les négociations (Hall, 1997; Palier et Surel, 2006). L'analyse des institutions, qui s'intéresse au «tissu plus ou moins ancien et serré de règles et de pratiques», replace les choix des acteurs dans une perspective plus large, en révélant comment les institutions régulent ces choix (Palier et Surel, 2006: 11). Enfin, l'analyse des idées s'attache à comprendre comment les «paradigmes, référentiels et les systèmes de croyances » entrent en compte dans les choix individuels ou collectifs (ibid.: 16), tout choix étant soustendu par des valeurs, un diagnostic du problème et un objectif recherché.

L'apport de l'approche des «trois I» est de montrer comment ces dimensions s'articulent, comme présenté en figure 1. Dans une logique de justification, les acteurs peuvent abandonner le registre de leurs intérêts et se référer à un principe supérieur. Le débat aboutit alors à la convergence sur un principe supérieur commun ou à l'affrontement de plusieurs principes (Boltanski et Thévenot, 1991). Le recours aux idées peut ainsi avoir pour finalité la légitimation des intérêts. Les institutions sont le lieu d'expression, puis d'arbitrages entre des intérêts divergents (Heclo, 1994). Enfin, les institutions relayent et hiérarchisent les idées contradictoires, mais elles sont aussi des espaces de délibération qui vont avoir un pouvoir reconstructif ou transformatif des idées, contribuant ainsi à l'élaboration de normes (Heclo, 1994).

Ce cadre d'analyse des "trois I» nous semble pertinent pour comprendre les jeux d'acteurs liés à l'évolution des paysages de référence. Quand la fonction fédératrice des paysages de référence est fragilisée, les antagonismes entre intérêts deviennent visibles. Nous allons mobiliser la notion de SE pour mettre en lumière ces intérêts 
divergents, et voir comment l'évolution des paysages va à l'encontre de certains intérêts. L'évolution des paysages donne lieu à de nouvelles interactions entre SE, qui s'opèrent dans le cadre d'institutions. Nous montrons comment les institutions en place régulent ces interactions, et en quoi ces changements récents peuvent les fragiliser. Enfin, l'évolution des paysages de référence affaiblit le consensus apparent sur l'idée que ces paysages sont l'expression d'une relation harmonieuse entre l'humain et la nature. Nous verrons comment ces désaccords s'expriment et sont mobilisés par les acteurs dans leur stratégie.

\subsection{Méthode d'enquête et d'analyse}

Le dispositif de terrain que nous avons déployé s'appuie à la fois sur des entretiens individuels et sur l'observation participante.

Nous avons réalisé 46 entretiens individuels semi-directifs entre juillet 2016 et août 2017, afin de recueillir une diversité de représentations sur les dynamiques paysagères du mont Lozère. Pour ce faire, nous avons cherché à rencontrer des individus aux profils variés en termes d'activités (Tableau 1), d'âge, de genre, de lieu de vie ou de parcours migratoire dans une optique de triangulation des informations (Olivier De Sardan, 2008). La phase d'enquête a abouti à la saturation des données.

Tableau 1. Activités des personnes rencontrées

\begin{tabular}{|c|c|c|c|}
\hline & Nombre d'individus ren & contrés & \\
\hline & & Éleveur bovin viande & 6 \\
\hline & & Éleveur bovin & 3 \\
\hline & & Berger/Éleveur ovin & 6 \\
\hline & & Éleveur caprin & 1 \\
\hline & & Apiculteur & 1 \\
\hline & & Conseiller & 2 \\
\hline Activité & & Forestier secteur privé & 3 \\
\hline & & Forestier secteur public & 5 \\
\hline & Tourisme & Prestataire touristique & 6 \\
\hline & & Technicien/Ingénieur & 5 \\
\hline & Environnement et chasse & Naturaliste & 1 \\
\hline & Élus & & 5 \\
\hline & & Pompier & 1 \\
\hline & & Artisan & 1 \\
\hline
\end{tabular}


Total

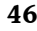

Une grille d'entretien a été établie en amont, avec des questions sur le parcours de vie, les pratiques et l'évolution des paysages. Le terme de SE n'a pas été utilisé dans les entretiens, mais il a été mobilisé comme une grille de lecture dans l'analyse. Les entretiens, d'une durée moyenne de $1 \mathrm{~h} 45$, ont été enregistrés et intégralement retranscrits.

20 Ces entretiens ont été complétés par l'observation participante du séminaire de terrain du Conseil scientifique (CS) du PNC (2 jours, août 2017), qui portait sur « les paysages et leur évolution en lien avec les pratiques agropastorales », et qui a réuni 16 chercheurs du CS, 18 agents du PNC et 5 invités. Les notes et les documents supports à cette réunion ont complété notre matériau d'analyse.

21 Lors de l'analyse, nous avons d'abord recensé les SE et disservices mentionnés dans les entretiens. Puis, pour les SE directement en lien avec le dérochage et le retournement de prairies, nous avons identifié, dans les entretiens et durant le séminaire du CS, les fournisseurs et les bénéficiaires des SE, ainsi que les acteurs intermédiaires. Enfin, nous avons caractérisé les intérêts de ces acteurs, les institutions qui régulent les interactions entre SE et les différentes idées des relations entre l'humain et la nature.

\section{Résultats}

\subsection{Analyse des intérêts : comment l'augmentation du dérochage et du retournement de prairies redessine le jeu d'interdépendances entre les acteurs?}

22 Si les pratiques du dérochage et du retournement de prairie font débat, c'est parce qu'elles sont centrales dans les stratégies des acteurs, soit comme un levier, soit comme un risque.

\subsubsection{Perceptions des différents types de milieux ouverts}

23 Nous avons recensé les SE des paysages ouverts mentionnés dans les entretiens, puis nous les avons classés selon le type d'écosystème (parcours, prairies permanentes, prairies temporaires). 
Figure 2. SE des paysages ouverts mentionnés dans les entretiens, par type d'écosystème

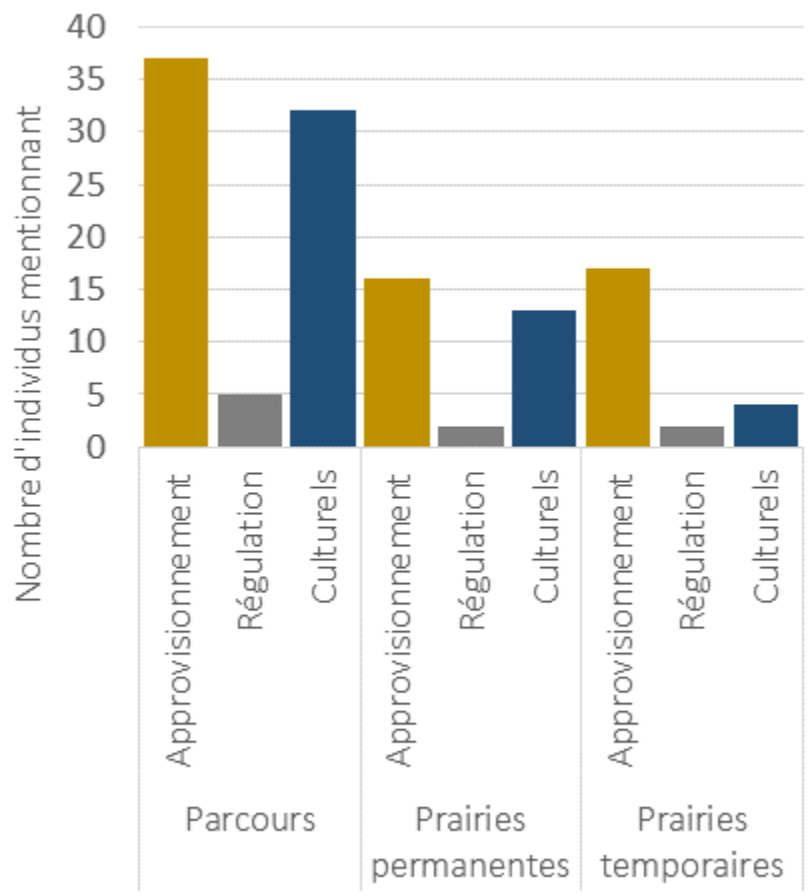

Comme le montre la figure 2, les parcours sont perçus comme les écosystèmes les plus importants en termes de SE : ils fournissent non seulement des SE d'approvisionnement (pour 37 individus) comme l'herbe, et des SE culturels (pour 32 individus), comme la valeur esthétique des paysages ouverts, l'existence de la biodiversité ou encore les activités de loisirs. Cette synergie est mise en évidence dans les entretiens : «Là-haut, qu'est-ce que vous voulez faire de mieux? De l'eau y en a, y a l'attrait touristique pendant la transhumance... c'est un joyeux équilibre. » (Éleveur ovin 1, EI ${ }^{1}$ ).

Les prairies permanentes et temporaires ne fournissent pas les mêmes SE : les prairies temporaires sont perçues comme fournissant un fourrage plus abondant et plus qualitatif. Ce point peut cependant faire l'objet de débats. Certains naturalistes mettent ainsi en avant la richesse nutritionnelle du foin produit par les prairies naturelles. Dans l'ensemble, les prairies permanentes sont davantage perçues comme fournissant des SE culturels (13 individus) que les prairies temporaires (4 individus). Les SE de valeur d'existence de la biodiversité sont les plus cités (7 individus).

Les paysages ouverts ne sont donc pas uniformes et sont perçus différemment par les acteurs.

Les pratiques de dérochage et de retournement de prairies modifient les paysages ouverts et les SE qu'ils fournissent. Le dérochage ne concerne en général que les parcours - les prairies ayant déjà été dérochées dans le passé - et est accompagné d'un labour, appelé retournement et conduisant à la mise en place d'une prairie temporaire. Les éleveurs peuvent aussi décider de retourner une prairie permanente, qui devient alors prairie temporaire. La figure 3 illustre l'impact de ces pratiques sur les paysages : la multiplication des pratiques de dérochage et de retournement de prairies implique une transition de paysages ouverts patrimoniaux où les parcours et les prairies permanentes dominent, à des paysages ouverts plus productifs, où la composante 
agricole, notamment les prairies temporaires, s'affirme. Ces pratiques agricoles impactent alors les $\mathrm{SE}$ : le dérochage et le retournement de prairies améliorent la quantité de foin, mais ont un impact négatif sur les SE d'approvisionnement (herbe, miel), les SE culturels (esprit des lieux, activités de loisirs et de sport, valeur esthétique et patrimoniale des paysages, existence de la biodiversité) et les SE de régulation (régulation de l'érosion, qualité de l'eau). Cette figure permet aussi d'identifier trois types d'acteurs : les fournisseurs/bénéficiaires de SE (les éleveurs); les bénéficiaires de SE (les apiculteurs, professionnels touristiques et touristes, le PNC, les habitants et citoyens, les naturalistes); et les acteurs intermédiaires (les agents du PNC, les élus).

Figure 3. Impact du dérochage et du retournement de prairies sur les SE et les acteurs

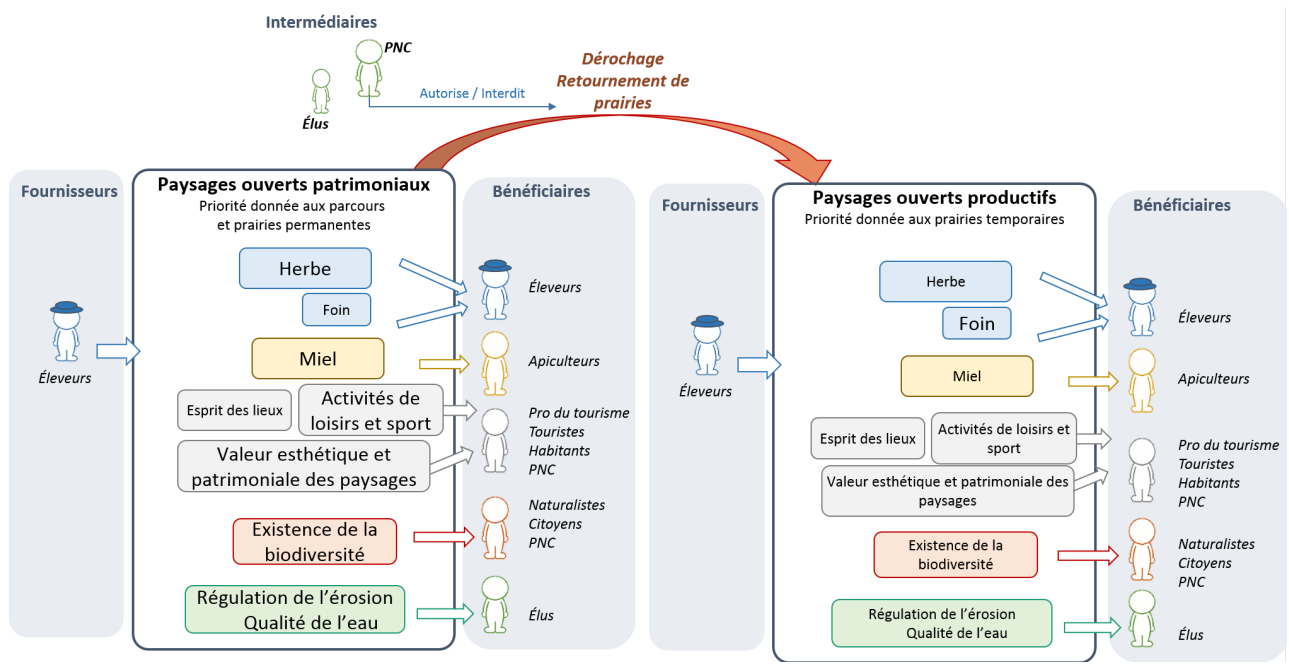

\subsubsection{Les éleveurs : fournisseurs et bénéficiaires de SE}


donc la création de nouvelles prairies de fauche.

"Du dérochage, peut-être que j'en ferai d'autres, dans le souci d'améliorer l'autonomie fourragère, ou de la conforter, du moins. » (Éleveur mixte 1, EI).

Des effets pervers de la PAC peuvent aussi stimuler ces pratiques de dérochage (par exemple, la soustraction des cailloux du calcul des surfaces primables incite au dérochage).

Enfin, le retournement de prairies pour transformer une prairie permanente en une prairie temporaire permet d'augmenter les rendements en foin.

\subsubsection{Les professionnels touristiques, les touristes, les naturalistes, les apiculteurs, les citoyens : bénéficiaires de SE}

Ces acteurs bénéficient des SE des paysages ouverts, qui sont coproduits par l'écosystème et les pratiques des éleveurs, par le biais d'une valorisation économique (développement d'une activité touristique, récolte de miel), ou d'activités non marchandes (randonnée, observation naturaliste...).

Les éléments abiotiques, tels les cailloux, fournissent aussi des SE culturels comme la valeur esthétique et patrimoniale :

"Chaque fois que je franchis le col, je m'arrête, je regarde les blocs de granite. Ça, on peut pas s'en passer, c'est un truc viscéral, on s'y attache. » (Élu 3, EI).

Le dérochage et le retournement de prairies apparaissaient pour ces acteurs comme un risque de dégradation des SE dont ils sont bénéficiaires et dont ils dépendent.

"On enlève les boules de plusieurs tonnes. Le côté mystérieux et emblématique du lieu, on est en train de le changer. » (Prestataire touristique 3, EI).

Dans cette citation, cette personne exprime la crainte de voir se dégrader la qualité du SE de valeur patrimoniale fournie par les blocs de granite, dont elle bénéficie à titre professionnel, mais aussi personnel. Face à ce qu'ils considèrent comme un risque, ces acteurs peuvent adopter un rôle d'alerte auprès d'acteurs plus influents.

\subsubsection{Les agents du Parc national et les élus : des acteurs intermédiaires}

Les acteurs intermédiaires régulent les pratiques des fournisseurs de SE et facilitent les arbitrages entre bénéficiaires de SE. Les élus sont concernés par la problématique du retournement de prairies dans la mesure où cela peut porter atteinte à la qualité de l'eau du fait de l'amendement, mais aussi car ces pratiques peuvent modifier les paysages de leur commune.

Le PNC est mandaté par l'État pour préserver, au nom de l'intérêt général, l'équilibre entre la conservation de la diversité biologique, la poursuite du développement économique et la sauvegarde des valeurs culturelles. Dans un contexte de fermeture des paysages, le PNC vise à maintenir une activité agricole au sein du territoire, notamment la présence de troupeaux qui pâturent les parcours et freinent les dynamiques d'enfrichement et de boisement spontané. À cet égard, le dérochage et le retournement de prairies, qui peuvent être indispensables à la survie des exploitations, peuvent être considérés comme nécessaires au maintien des paysages ouverts.

«Du moment qu'on autorise et que l'on déclare comme prioritaire le maintien des gens en activité sur ce territoire, il est clair qu'il faut en accepter les effets sur le paysage et qu'il faut même en conserver les traces... » (Agent du PNC 1, CS²). 

PNC en vertu des textes réglementaires (code de l'environnement, charte). La procédure suit cinq étapes : la demande de l'agriculteur réalisée avec un agent du PNC; le rapport d'instruction complété par le technicien agri-environnement avec avis et prescriptions ; la demande d'avis interne, complétée par les différents services du PNC ; l'avis du CS et enfin l'arrêté signé qui rappelle les articles de lois faisant référence, octroie ou non l'autorisation de travaux assortie de recommandations. De janvier 2015 à fin août 2018, à l'échelle du PNC, 66 arrêtés avaient été accordés pour des travaux agricoles (dont dérochage et retournements de prairies), pour seulement une demande refusée.

\subsubsection{Le système d'autorisations : la recherche d'un compromis entre SE antagonistes}

Dans la procédure d'instruction des demandes de travaux, l'effet des pratiques agricoles sur les différents SE est évalué par l'agent instructeur, les services du PNC et par les membres du CS. Par exemple, dans le cas d'une demande de dérochage et de retournement de prairies, les agents du PNC émettent l'avis suivant:

"Pour le dérochage, le problème est sensible, nous sommes sur un lieu extrêmement fréquenté du massif, en toute saison, avec un pic à la belle saison " (Agent du $\mathrm{PNC}, \mathrm{AI}^{3}$ ).

Dans cette procédure d'instruction, l'effet des pratiques agricoles sur les différents SE, comme l'activité de randonnée, est pris en compte de façon détaillée, en tenant compte

Développement durable et territoires, Vol. 10, n² | Juillet 2019 
des spécificités du lieu et du projet de l'éleveur. Les recommandations en termes de pratiques témoignent de la recherche d'un compromis entre les SE antagonistes, ici les SE de foin et les SE de la valeur patrimoniale des paysages.

"Une technique serait de dérocher superficiellement la parcelle, en extrayant les blocs les plus visibles, et de casser les têtes des gros blocs très enterrés. » (Agent du PNC, AI).

\subsubsection{Quelle prise en compte des interactions entre SE à l'échelle du paysage et sur le temps long?}

En règle générale, les autorisations sont données de façon ponctuelle, à l'échelle de la parcelle. De fait, l'effet cumulé de ces pratiques ne se constate qu'a posteriori.

"Des autorisations de travaux ont été données entre 2000 et 2006, et ce n'est qu'aujourd'hui qu'on voit les effets sur le paysage.» (Membre du CS).

Cette citation montre que c'est la multiplication de pratiques ponctuelles dans l'espace qui a un effet à l'échelle du paysage et qui peut aboutir à la dégradation des SE, ici la valeur patrimoniale des paysages ouverts. Ces pratiques peuvent avoir un caractère irréversible, entraînant la perte définitive de certains SE. Or, les autorisations sont données ponctuellement, à l'échelle de la parcelle, ce qui ne permet pas de prendre en compte les effets des pratiques à l'échelle paysagère et sur le temps long. L'augmentation des demandes d'autorisations de dérochage et de retournement de prairies renforce le risque d'effet cumulatif et d'irréversibilité, et explique que le PNC s'interroge sur les limites du système actuel.

\subsection{Analyse des idées : comment les acteurs intègrent les pratiques de dérochage et le retournement de prairies dans l'histoire des relations entre l'humain et la nature?}

La compréhension des jeux d'acteurs autour des questions de dérochage et du retournement de prairies ne peut se limiter à l'analyse des intérêts et des institutions. Le discours renvoie aussi à une dimension normative, et intègre les pratiques de dérochage et de retournement de prairies dans l'histoire des relations entre les humains et la nature.

\subsubsection{Dérocher, retourner les prairies : des améliorations dans la continuité du travail des anciens}

Un premier type de discours, porté par les agriculteurs et les conseillers agricoles, insiste sur l'ancienneté des pratiques de dérochage et de retournement de prairies, qui s'inscrivent dans la continuité du travail des anciens. Cependant, le dérochage était réalisé dans des modalités différentes du dérochage actuel : il était manuel, donc les bocs étaient plus petits; et ils étaient regroupés au sein des parcelles plutôt qu'évacués.

Le terme « améliorer » revient souvent pour décrire ces pratiques :

"On a amélioré les terres, surtout au niveau des prés de fauche. Il y avait beaucoup de cailloux, des endroits humides qu'on a fumés, qu'on a dérochés. " (Éleveur mixte 1, EI).

Le terme d'amélioration traduit pour ces acteurs la synergie entre l'humain et la nature, puisque les pratiques agricoles permettent de valoriser un potentiel naturel. La modification des moyens techniques, comme le recours à une pelle mécanique pour le 
dérochage, s'inscrit dans une logique de progrès inhérente à l'histoire de l'agriculture. Ainsi, dans ce premier type de discours, les relations entre l'humain et la nature sont marquées par deux constantes : le caractère fortement anthropisé des paysages ouverts et l'évolution continue des moyens techniques des agriculteurs. Le dérochage ou le retournement de prairies tels qu'ils sont pratiqués aujourd'hui s'inscrivent dans cette continuité.

\subsubsection{Le dérochage et le retournement : le risque d'une rupture dans les relations entre l'humain et la nature}

51 Dans un second type de discours, porté par quelques prestataires touristiques, naturalistes, ainsi que par certains agents du PNC, le retournement de prairies, et surtout le dérochage, sont décrits comme irréversibles :

"Ce qu'il y a de plus choquant, c'est que l'on ne garde pas la mémoire des actions de l'homme. On ne reconnaît plus sur le mont Lozère la granularité du terrain, les amas. On perd la rareté, la spécificité géologique. » (Agent du PNC 2, CS).

Dans cette citation, on voit que le dérochage tel qu'il est pratiqué aujourd'hui, est considéré comme un risque, car il rend invisibles les traces des anciennes pratiques et les conditions naturelles. Ainsi, les pratiques de dérochage sont identifiées comme un risque majeur de rupture, lié à la mécanisation et la capacité inédite qu'elle permet en termes de taille et tonnage des rochers à déplacer ou à détruire, de superficie de parcelles à transformer en un seul temps.

53 L'image stabilisée d'une synergie entre l'humain et la nature, visible dans les paysages ouverts, et surtout dans les parcours, se trouve selon ces acteurs remise en question.

\subsection{Lien entre les intérêts, les institutions et les idées}

\subsubsection{Intérêts et institutions : quelle prise en compte des intérêts antagonistes dans le système des autorisations?}

Le système d'autorisation vise à réguler les interactions entre $\mathrm{SE}$, en prenant en compte les différents intérêts des bénéficiaires de SE. L'arbitrage est complexe, car les intérêts, s'ils sont antagonistes, sont aussi reconnus comme légitimes, par exemple, l'intérêt de l'éleveur d'atteindre l'autonomie fourragère et l'impératif de préserver la valeur patrimoniale des paysages:

"Il est inquiétant de voir les demandes de dérochements se généraliser, ce qui entraîne une perte lente, mais inéluctable des paysages caractéristiques du mont Lozère. Malheureusement, la demande du pétitionnaire, soulignant son besoin d'autonomie fourragère, nous conduit à ne pas pouvoir refuser cette autorisation. » (Agent du PNC, AI).

Pour prendre en compte au mieux les différents intérêts, le système s'appuie sur deux mécanismes : le recours aux différents services du PNC (avis interne) et aux différentes disciplines scientifiques (avis du CS). Ce mécanisme suppose qu'ainsi l'ensemble des intérêts pourront être pris en compte. Cependant, certains intérêts peuvent être mal ou pas représentés au sein de cette institution.

"Le PNC autorise à labourer à 1400 mètres. J'étais scandalisé. Aujourd'hui, les élus peuvent faire partie du conseil d'administration, c'est terrible parce que les électeurs font pression sur l'élu. Nous, on n'est pas nombreux, notre façon de voir n'est pas du tout représentative..." (Prestataire touristique 3, EI). 
"Le classement Unesco et les parcs, ça pourrait être bien, mais faudrait qu'on les gère. On n'a rien à dire dans ces entités-là. » (Éleveur ovin 1, EI).

Pour des raisons différentes, l'un estimant que le rôle des élus est peu représentatif de l'intérêt général, l'autre considérant que les personnes locales n'ont pas assez de pouvoir dans les organismes de protection, ces personnes considèrent que leurs intérêts ne seront pas pris en compte dans l'arbitrage des antagonismes entre SE, dans un rapport de force qui leur est défavorable.

Ainsi, si le système d'autorisations consiste à arbitrer entre des intérêts contradictoires, les intérêts ne sont pas forcément tous représentés. Le terme d'arbitrage est également à nuancer, car le PNC n'est pas une institution neutre, elle est partie prenante dans le jeu d'acteurs.

\subsubsection{Intérêts et idées : comment les idées sont mobilisées pour asseoir la légitimité des acteurs à " faire du paysage » et servir leurs intérêts ?}

L'analyse montre que les acteurs n'évoquent pas les idées des relations entre l'humain et la nature de façon désintéressée. En décrivant les pratiques de dérochage et de retournement de prairies comme l'expression d'une relation ancienne et durable entre l'humain et la nature, les éleveurs s'érigent en garants des paysages :

"Si nos paysages sont beaux, c'est parce qu'on est dedans. Et parfois, c'est très, très dur! Il faut continuer à évoluer, on peut pas faucher à la faux comme les ancêtres!» (Éleveur ovin 1, EI).

Cette citation montre les ressorts de la légitimation des agriculteurs : le travail que ces pratiques représentent, l'effet positif de ces pratiques sur les paysages et la revendication d'une liberté de moyens face à l'objectif à atteindre.

60 À l'inverse, en exposant le risque de rupture dans les relations entre l'humain et la nature, d'autres acteurs affirment le rôle essentiel de prévention qu'ils ont sur le paysage, soit en alertant, soit en régulant. Ils ont recours à deux types de légitimation : la légitimation par la connaissance et la légitimation par la loi.

"Le citoyen lambda qui voit une prairie temporaire, qui fait du vert dans le paysage, c'est beau, ça change du goudron de la ville. Mais pour nous, c'est aussi pauvre que la ville... Personne n'a cette connaissance très fine de la nature. " (Naturaliste 1, EI).

61 Cette citation montre un exemple de légitimation par la connaissance : c'est parce qu'il a des connaissances que le naturaliste porte un regard initié sur la nature, il est donc légitime à agir sur le paysage, en donnant l'alerte.

La légitimation par la loi confère certains pouvoirs au PNC, qui est mandaté par l'État pour assurer des missions d'intérêt général. Il est donc habilité à encadrer les pratiques des agriculteurs en vertu de textes réglementaires.

Finalement, on voit que les idées des relations entre l'humain et la nature sont mobilisées dans une optique bien précise : légitimer l'action des différents acteurs sur le paysage, que ce soit celle de l'éleveur qui retourne une prairie, celle du militant naturaliste qui mène une action de sensibilisation ou celle de l'agent du PNC qui peut émettre des recommandations sur les pratiques à suivre. 


\subsubsection{Idées et institutions : quelle opérationnalisation d'une idée des relations entre l'humain et la nature à travers le système d'autorisations ?} paysages de référence, modifie les jeux d'acteurs, un premier niveau d'analyse consiste à dire que les espaces ouverts, auparavant supports de synergies, sont maintenant également des espaces d'antagonismes. En effet, les paysages ouverts, et en particulier les parcours, ont été décrits comme étant le support de synergies entre des SE de différentes catégories, mais aussi entre les intérêts de différents acteurs, dans une conception harmonieuse des relations entre l'humain et la nature. Les institutions, comme le PNC ou la reconnaissance par l'Unesco, viseraient à protéger ces synergies et solidarités écologiques (Mathevet et al., 2018). Les références à cette synergie sont nombreuses, à la fois dans les documents de gestion comme la charte du PNC (PNC, 2013), mais aussi dans les entretiens. Par opposition, l'augmentation des pratiques de dérochage et de retournement de prairies suscite de nouveaux antagonismes entre SE. D'autres jeux d'acteurs apparaissent, interrogeant tant le rôle des éleveurs dans le paysage que la capacité des institutions en place à répondre à ces nouveaux enjeux.

Cependant, il convient de se rappeler que l'image de paysages ouverts comme espaces de synergies est le résultat d'une évolution historique (Marty et Lepart, 2001). La 
présence des mêmes éléments dans les documents officiels et les discours individuels montre comment s'est opérée cette construction sociale : les institutions (ici au sens d'organisations) se font le relais des représentations communément partagées, mais elles ont également un rôle performatif pour stabiliser et diffuser ces représentations. C'est par ce mécanisme que les paysages ouverts ont été mobilisés comme un élément identitaire fort dans les projets de développement, pouvant guider l'action (PeyracheGadeau et Perron, 2010). Ce processus s'est fait au détriment de la reconnaissance de la diversité des représentations liées aux paysages ouverts, que nous avons vue dans les entretiens, et qui a également été décrite par d'autres auteurs (Guisepelli, 2005 ; Lazaro et Eychenne, 2012, Barnaud et al., 2015). Finalement, on voit que la construction des paysages ouverts comme paysage de référence est passée par la valorisation d'une certaine représentation du paysage, relayée par le PNC, masquant ainsi l'existence de divergence de représentations des paysages ouverts. Plutôt que de parler de nouveaux antagonismes dans les jeux d'acteurs, il faudrait donc évoquer une mise à jour d'antagonismes existants, qui avaient été masqués dans le processus de construction des paysages de référence, et des compromis nécessaires à la fabrique de la charte du territoire du PNC.

\subsection{Les paysages ouverts, paysages immuables?}

70 Par ailleurs, il est nécessaire de s'arrêter sur la question de la temporalité et du caractère dynamique des paysages de référence. Davodeau et al. (2018) ont montré comment diverses temporalités s'imbriquent dans la gestion du patrimoine ligérien et que les conflits entre acteurs peuvent s'expliquer par le fait qu'ils se réfèrent à différents moments de l'histoire du socio-écosystème. Dans le cas du mont Lozère, deux temporalités s'imbriquent: d'un côté, une dynamique lente et naturelle, celle de l'augmentation du couvert forestier, et de l'autre les dynamiques des paysages ouverts, évolutions anthropiques, rapides et dont les conséquences se verront à long terme. L'imbrication de ces deux temporalités aboutit au syndrome de glissement de référence (Pauly, 1995), c'est-à-dire le fait que l'état considéré comme qualitatif d'un milieu évolue dans le temps, et ce sans que les acteurs ne s'en aperçoivent, ce qui peut aboutir à tolérer la dégradation d'un milieu. Ainsi, on observe d'un côté une inertie des normes sur la question de la fermeture des paysages, où malgré l'augmentation du couvert forestier, les paysages ouverts sont toujours considérés comme les paysages de référence, et de l'autre un glissement de référence au sein des paysages ouverts, où on observe une acceptation des changements et de leurs conséquences sur la biodiversité.

Cependant, alors que dans les récits l'évolution de la forêt semble marquée par des jalons historiques datés, la description des paysages ouverts s'inscrit dans un «avant » sans repère : le PNC évoque dans sa charte des paysages "produits par des millénaires de pastoralisme et de transhumance» (PNC, 2013: 20), les éleveurs font référence aux " ancêtres ». Pour devenir un paysage de référence, il semble nécessaire non seulement de replacer ces paysages dans le temps long, mais aussi de l'extraire de la chronologie pour renvoyer à un âge d'or, qui tient plus de la mythologie que de l'histoire (Berque, 1996).

72 Finalement, le cas du mont Lozère permet de comprendre comment la fonction fédératrice des paysages et leur dimension temporelle se combinent. Les paysages ouverts font l'objet d'une double « codification » (Sgard, 2011) : loin de se référer à un 
moment précis de l'histoire du mont Lozère, les paysages de référence renvoient à une période idéalisée, où les relations entre l'humain et la nature seraient en synergie et, ce faisant, construisent un bien patrimonial à préserver. L'idéalisation extrait ces paysages de leur dimension historique et aboutit à les définir comme des paysages immuables. De fait, les évolutions récentes des paysages ouverts modifient cette conception de l'état de référence, en mettant en avant le caractère dynamique de ces paysages ainsi que la diversité d'usages et de pratiques sur ces paysages.

\subsection{Mettre en débat l'état de référence}

73 L'évolution des paysages de référence apparaît comme un angle mort de la gouvernance des paysages. Très présents dans la gouvernance des paysages, les états de référence sont mobilisés en tant qu'objet fédérateur, une finalité de développement communément partagée. Quand les paysages changent, les paysages de référence se trouvent objet de controverses, voire de conflits. Dans un tel contexte, il est nécessaire d'accepter de considérer les paysages de référence comme un objet de débat, non pas comme un passé idéalisé, mais comme le support d'une discussion sur les modèles de développement du territoire. C'est dans cet objectif que, suite au travail d'analyse décrit dans cet article, nous avons conçu et animé un jeu de rôles nommé Secoloz, qui modélise les interactions socio-écologiques sur le mont Lozère (Moreau et al., 2019). Les séances de jeu de rôles, auxquelles ont participé des éleveurs, des agents du PNC et des élus du mont Lozère, ont permis de mettre en débat l'état de référence, en particulier sa dynamique dans le temps et l'espace, son rôle dans la gouvernance du territoire et les enjeux liés au glissement de l'état de référence (Moreau, 2019).

\section{Conclusion} des paysages ouverts (Marty et Lepart, 2001; Le Floch et Devanne, 2003) et de la négociation entre les acteurs, autour de la charte du PNC par exemple. Par ailleurs, ce cas d'étude montre les tensions qui peuvent exister entre un état de référence conçu comme un objectif de gestion des paysages, dans une optique de conservation ou de restauration, et un état de référence mobilisé comme un cadre pour situer l'action. Finalement, l'état de référence oscille entre un rôle normatif et un rôle descriptif, et confond souvent un état historique méconnu, idéalisé et rémanent et un idéal futur (Donadieu, 2002). Ce flou qui entoure l'état de référence lui permet d'ailleurs de jouer ce rôle fédérateur dans la gouvernance des paysages, dans la mesure où il est mobilisé de façon implicite et va servir de support à l'expression des désirs et des craintes variés, voire incompatibles (Davodeau et Barraud, 2018).

La question du rôle de l'état de référence dans la gouvernance des paysages se pose de façon exacerbée dans les parcs nationaux. En effet, depuis la loi de réforme des parcs nationaux en 2006, ces derniers sont invités à penser, via la concertation avec les parties prenantes, les interdépendances sociales et écologiques et les solidarités associées (Mathevet et al., 2010) puis à définir leur "caractère » et les principes de gestion du territoire dans une charte. L'exemple du mont Lozère montre que le besoin de mettre en débat les interdépendances et cet état de référence reste crucial. Au final,

Développement durable et territoires, Vol. 10, n² | Juillet 2019 
ce travail souligne tout l'intérêt de développer des dispositifs de recherche-action, à la fois participatifs et pluridisciplinaires, pour analyser cette dynamique et pour accompagner les acteurs dans le pilotage des changements paysagers et territoriaux.

\section{BIBLIOGRAPHIE}

Barnaud C., Antona. M., 2014, « Deconstructing ecosystem services : Uncertainties and controversies around a socially constructed concept ", Geoforum, vol. 56, n 0, p. 113-123, https:// www.sciencedirect.com/science/article/pii/S0016718514001547.

Barnaud C., Corbera E., Muradian R., Salliou N., Sirami C., Vialatte A., Choisis J.-P., Dendoncker N., Mathevet R., Moreau C., Reyes-García V., Boada M., M. Deconchat M., Cibien C., Garnier S., Maneja R., Antona M., 2018, « Ecosystem Services, Social Interdependencies, and Collective Action : A Conceptual Framework », Ecology and Society, vol. 23, n 1, https://www.ecologyandsociety.org/ vol23/iss1/art15/.

Barnaud C., Theil L., Choisis J.-P., Eychenne C., 2015, « Les services écosystémiques : une notion savante déconnectée des représentations locales? Une analyse des représentations de l'élevage en zone agricole défavorisée ", in Environnement, politiques publiques et pratiques locales, édité par P. Beringuier, F. Blot, B. Desailly, M. Saqalli, Paris, L'Harmattan.

Bennett E. M., Peterson G. D., Gordon L. J., 2009, « Understanding relationships among multiple ecosystem services », Ecol Lett 12 (12), p. 1394-1404, https://onlinelibrary.wiley.com/doi/full/ 10.1111/j.1461-0248.2009.01387.x.

Berbés-Blázquez M., González J. A., Pascual U., 2016, « Towards an ecosystem services approach that addresses social power relations », Current Opinion in Environmental Sustainability, vol. 19, p. 134-43, https://www.sciencedirect.com/science/article/pii/S1877343516300070.

Berque A., 1994, Cinq propositions pour une théorie du paysage, Seyssel, Champ Vallon.

Berque A., 1996., Être humains sur la terre, Paris, Gallimard, coll. « Le débat ».

Boltanski L., Thévenot L., 1991, De la justification : les économies de la grandeur, Paris, Gallimard.

Burellier F., Baudry J., 1999. Écologie du paysage, Concepts, méthodes et applications, Paris, Tec \& Doc Lavoisier.

CE, 2000, « Convention européenne du paysage », Signée à Florence, Italie, le 20 octobre 2000.

Davodeau H., 2007, «Éclairer la boîte noire du paysage », Journées INRA-SFER de recherches en sciences sociales, Paris.

Davodeau H., Barraud R., 2018, « Quelle position adopter face au patrimoine qui vient ? La médiation paysagère pour mettre en débat les temporalités ", Développement durable \& territoires, vol. 9, n 2, https://journals.openedition.org/developpementdurable/12277.

Deuffic P., 2005, « La fermeture des paysages dans le Massif central : regards d'habitants sur une question d'experts », Cahiers d'économie et sociologie rurales, $n^{\circ}$ 75, p. 75-96.

Donadieu P., 2009, Les paysagistes : ou les métamorphoses du jardinier, Arles, Actes Sud Nature. 
Donadieu P., 2002, «Les références en écologie de la restauration », Revue d'écologie, supplément $n^{\circ}$ 9, p. 109-120, http://documents.irevues.inist.fr/handle/2042/55525.

Felipe-Lucia M., Martin-Lopez B., Lavorel S., Berraquero-Diaz L., Escalera-Reyes J., Comin F. A., 2015, « Ecosystem Services Flows : Why Stakeholders' Power Relationships Matter », PLoS One vol. 10, n 7, https://journals.plos.org/plosone/article?id=10.1371/journal.pone.0132232.

Filoche G., 2007, « La réforme des parcs nationaux français. Diversification des acteurs, redéfinition des compétences et des outils de gestion ", Revue européenne de droit de l'environnement, vol. 11, n 3, p. 309-320, https://www.persee.fr/doc/ reden_1283-8446_2007_num_11_4_1983.

Friedberg C., Cohen M., Mathieu N., 2000, « Faut-il qu'un paysage soit ouvert ou fermé ? L'exemple de la pelouse sèche du causse Méjan ", Nature Sciences Sociétés, vol. 8, n 4, p. 26-42, https://www.nss-journal.org/articles/nss/abs/2000/04/nss20000804p26/nss20000804p26.html.

Guisepelli E., 2005, « Les représentations sociales du paysage comme outils de connaissance préalable à l'action. L'exemple des Alpes du Nord », Cybergeo : European Journal of Geography, https://journals.openedition.org/cybergeo/3352.

Hall P., 1997, « The Roles of Interests, Institutions, and Ideas in the Comparative Political Economy of the Industrialized Nations ", in M. Irving Lichbach, A. S. Zuckerman, Comparative Politics : Rationality, Culture, and Structure, Cambridge, Cambridge University Press.

Heclo H., 1994, «Ideas, Interests and Institutions », in C. Dodd Lawrence et C. Jillson Calvin, The Dynamics of American Politics : Approaches and Interpretations, Boulder, Westview Press.

Hervieu B., Purseigle F., 2013, Sociologie des mondes agricoles, Paris, Armand Colin.

Labat D., Aggeri G., 2013, « La loi Paysage a-t-elle eu un impact sur la planification territoriale ? ", Projets de paysage, $\mathrm{n}^{\circ}$ 9, https://www.projetsdepaysage.fr/

la_loi_paysage_a_t_elle_eu_un_impact_sur_la_planification_territoriale_.

Lamarque P., Tappeiner U., Turner C, Steinbacher M, Bardgett R., Szukics U., Schermer S., Lavorel S., 2011, «Stakeholder perceptions of grassland ecosystem services in relation to knowledge on soil fertility and biodiversity », Regional Environmental Change, Manque : Volume 11, Issue 4, p 791-804, https://doi.org/10.1007/s10113-011-0214-0.

Larrère R., Lizet B., Berlan-Darqué M., 2009, Histoire des parcs nationaux : comment prendre soin de la

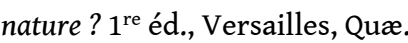

Lazaro L., Eychenne C., 2012, « Le paradoxe du paysage, un “consensus équivoque” ». L'exemple du pastoralisme collectif pyrénéen et de son rôle sur le paysage montagnard », Les Agriculteurs, Acteurs du paysage, La Bastidonne, https://hal.archives-ouvertes.fr/hal-01627122.

Le Floch S., Devanne A.-S., Deffontaines J.-P., 2005, « La « fermeture du paysage » : au-delà du phénomène, petite chronique d'une construction sociale ", L'Espace géographique vol. $34, \mathrm{n}^{\circ} 1$, p. 49-64, https://www.cairn.info/revue-espace-geographique-2005-1-page-49.htm.

Le Floch S., Devanne A.-S., 2003, Qu'entend-on par « fermeture du paysage? », Cemagref.

Lepart J., Marty P., Rousset O., 2000, « Les conceptions normatives du paysage. Le cas des Grands Causses », Nature Sciences Sociétés, vol. 8, n 4, p. 15-25, https://doi.org/10.1016/

S1240-1307(01)80003-0.

Luginbühl Y., 2007, « Pour un paysage du paysage », Économie rurale. Agricultures, alimentations, territoires, $\mathrm{n}^{\circ}$ 297-298, p. 23-37. 
Marty P., Lepart J., 2001, « Forêts et milieux ouverts : anciennes et nouvelles légitimités », Bulletin de l'association géographique française, $\mathrm{n}^{\circ}$ 78-2, p. 177-89, https://www.persee.fr/doc/ bagf_0004-5322_2001_num_78_2_2216.

Mathevet R., Thompson J., Delanoë O., Cheylan M., Gil-Fourrier C., Bonnin M., 2010, « La solidarité écologique : un nouveau concept pour une gestion intégrée des parcs nationaux et des territoires ", Natures Sciences Sociétés, vol. 18, n 4, p. 424-433, https://www.cairn.info/revuenatures-sciences-societes-2010-4-page-424.htm.

Mathevet R., Bousquet F., Larrère C., Larrère R., 2018, « Environmental Stewardship and Ecological Solidarity : Rethinking Social-Ecological Interdependency and Responsibility ", Journal of Agricultural and Environmental Ethics, vol. 31, $n^{\circ} 5$, https://link.springer.com/article/ 10.1007 \%2Fs10806-018-9749-0.

MEA, 2005, Ecosystems and human well-being, vol. 5, Island press Washington, DC.

Moreau C., 2019, Mettre en débat l'état de référence. Analyse des représentations des dynamiques paysagères au prisme des services écosystémiques. L'exemple du mont Lozère, thèse de l'université de Montpellier.

Moreau C., Barnaud C., Mathevet R., 2019, « Conciliate Agriculture with Landscape and Biodiversity Conservation : A Role-Playing Game to Explore Trade-Offs among Ecosystem Services through Social Learning », Sustainability, vol. 11, n² 2, p. 310.

Olivier De Sardan J.-P., 2008, La rigueur du qualitatif : les contraintes empiriques de l'interprétation socio-anthropologique, Louvain-la-Neuve, Academia-Bruylant.

Palier B., Surel Y., 2006, « Les "trois I" et l'analyse de l'État en action », Revue française de science politique, vol. $55, \mathrm{n}^{\circ} 1$, p. 7-32, https://www.cairn.info/revue-francaise-de-sciencepolitique-2005-1-page-7.htm.

Pauly D., 1995, « Anecdotes and the shifting baseline syndrome of fisheries », Trends in ecology \& evolution, vol. $10, \mathrm{n}^{\circ} 10$, p. 430.

Perrier-Cornet P., 2002, Repenser les campagnes, La Tour-d'Aigues, Éd. de l'Aube.

Peyrache-Gadeau V., Perron L., 2010, « Le paysage comme ressource dans les projets de développement territorial ", Développement durable \& territoires, vol. 1, $\mathrm{n}^{\circ} 2$, https:// journals.openedition.org/developpementdurable/8556.

PNC, 2013, « La charte du Parc national des Cévennes », Florac, PNC.

Sgard A., 2011, Le partage du paysage, thèse d'habilitation à diriger les recherches, université de Grenoble.

Shackleton C. M., Ruwanza S., Sinasson Sanni G K., Bennett S., De Lacy P., Modipa R., Mtati N., Sachikonye M., Thondhlana G., 2016, « Unpacking Pandora's Box : Understanding and Categorising Ecosystem Disservices for Environmental Management and Human Wellbeing ", Ecosystems, vol. 19, n 4, p. 587-600, https://doi.org/10.1007/s10021-015-9952-z.

\section{NOTES}

1. Entretien individuel.

2. Séminaire de terrain du Conseil scientifique.

3. Avis d'instruction. 


\section{RÉSUMÉS}

Les paysages de référence participent de la gouvernance des paysages. Ainsi, sur le mont Lozère, face à la fermeture des paysages, les institutions en place visent à préserver les paysages ouverts qui sont considérés comme des paysages de référence. Dans cet article, nous analysons la façon dont l'évolution des paysages ouverts, sous l'effet de l'évolution des pratiques agricoles, modifie les jeux d'acteurs et fragilise la gouvernance territoriale. Nos résultats montrent que l'évolution des paysages de référence reste un angle mort dans la gouvernance des paysages : mobilisés en tant qu'élément fédérateur, les paysages de référence sont présentés comme consensuels et immuables, ce qui tend à masquer la diversité des représentations dont ils font l'objet, ainsi que leur caractère dynamique. Ce cas d'étude invite à considérer les états de référence comme un objet de débat.

Baseline landscapes play a part in the landscape governance. On the Mont Lozere, France, the institutions aim to preserve open landscapes, which are considered as baseline landscapes. In this paper, we analyse how the open landscape dynamics due to the evolution of farming practices impact the stakeholder interplay and weaken the local governance. Our results show that the evolution of baseline landscapes is a blind spot in the landscape governance. Baseline landscapes are used in the development project as a unifying element, considered as consensual and static. This masks the diversity of representations about baseline landscapes and their dynamics. This case study advocates for considering baseline landscapes as a subject of debate.

\section{INDEX}

Mots-clés : paysage, aire protégée, gouvernance, service écosystémique, état de référence, jeux d'acteurs, mont Lozère

Keywords : landscape, protected area, governance, ecosystem service, baseline, stakeholder analysis, Mont Lozere

\section{AUTEURS}

\section{CLÉMENCE MOREAU}

Clémence Moreau est docteure en géographie. Elle travaille sur les jeux d'acteurs liés aux enjeux environnementaux comme les paysages.

Dynafor, université de Toulouse, INPT, INRA, Toulouse.

clemencemoreau0@gmail.com

\section{CÉCILE BARNAUD}

Cécile Barnaud est chercheuse en géographie humaine. Elle travaille sur les interactions sociales (conflits, négociations, collaborations) à l'interface agriculture-société-environnement à l'échelle des territoires.

Dynafor, université de Toulouse, INPT, INRA, Toulouse.

cecile.barnaud@inra.fr 


\section{RAPHAËL MATHEVET}

Raphaël Mathevet est écologue et géographe. Il travaille sur la conservation de la biodiversité, la gestion concertée des aires protégées et des usages multiples des ressources naturelles.

CEFE, CNRS, université de Montpellier, université Paul-Valéry Montpellier 3, EPHE, IRD

Montpellier, France, et Institut français de Pondichéry, UMIFRE 21 CNRS-MAEE/USR 3330, Inde.

raphael.mathevet@cefe.cnrs.fr 